\title{
La experiencia de la modernización administrativa en Portugal. Algunas lecciones para las instituciones públicas españolas post-Covid-19
}

\section{The experience of administrative reform in Portugal. Some lessons for post-Covid-19 spanish public institutions}

\author{
Enrique José Varela Álvarez \\ Universidade de Vigo (España) \\ ORCID: http://orcid.org/0000-0001-9021-0797 \\ evalvarez@uvigo.es
}

\section{NOTA BIOGRÁFICA}

Profesor de Ciencia Política y de la Administración de la Universidade de Vigo. Decano de la Facultad de Dirección y Gestión Pública y coordinador académico del Observatorio de Gobernanza $\mathrm{G}^{3}$ de la misma Universidad, es autor de numerosas publicaciones en el campo de la gobernanza y la cooperación institucional, así como en la dirección, la gestión y el liderazgo público en las instituciones centrales, autonómicas y locales españolas, además de en los espacios institucionales multinivel y transfronterizos de la Unión Europea.

\section{RESUMEN}

El reto de la modernización del Estado sigue estando presente en las agendas de todos los países que tienen que enfrentar desafíos como la actual pandemia de COVID-19. En el caso de España y Portugal, encontramos una larga tradición de procesos de reformas y modernizaciones administrativas desde la segunda mitad del siglo $x x$ hasta nuestros días. En el trabajo que se presenta a continuación se revisa la experiencia de modernización del Estado portugués con el objetivo puesto en el aprendizaje de su «senda de dependencia» institucional (de la «persistencia institucional», hasta la «innovación incremental»), con el fin de poder aprender de sus aciertos y errores político-administrativos y conseguir, así, mejorar el conocimiento sobre los proyectos de cambio administrativo de la Administración General del Estado, en el contexto de los planes de recuperación y resiliencia de la Comisión Europea y del Gobierno de España.

\section{PALABRAS CLAVE}

Reforma administrativa; isomorfismo institucional; innovación incremental; innovación pública.

\begin{abstract}
The challenge of modernizing the State continues to be on the agendas of all countries that have to face threats such as the current COVID-19 pandemic. In the case of Spain and Portugal, we find a long tradition of administrative reform and modernization processes from the second half of the 20th century to the present day. In the work presented below, the Portuguese State expirience reform is reviewed with the aim of learning its institutional "path dependence» (from «institutional persistence» to «incremental innovation»), in order to be able to learn from their political and administrative successes and errors and thus improve knowledge about the administrative change projects of the Central State Administration, in the context of the recovery and resilience plans of the European Commission and the Government from Spain.
\end{abstract}

\section{KEYWORDS}

Administrative reform; institutional isomorphism; incremental innovation; public innovation. 
DA. Nueva Época - N. ${ }^{\circ}$, enero-diciembre 2020 - ISSN: 1989-8983 - DOI: https://doi.org/10.24965/da.i7.10897 - [Págs. 145-155]

La experiencia de la modernización administrativa en Portugal. Algunas lecciones para las instituciones públicas..

Enrique José Varela Álvarez

\begin{abstract}
SUMARIO
PROEMIO: ESPAÑA 2020, ¿POR QUÉ APENAS HEMOS APRENDIDO TRAS CINCUENTA AÑOS DE REFORMAS DEL ESTADO? I. EL SISTEMA POLÍTICO-ADMINISTRATIVO PORTUGUÉS, TAN LEJOS TAN CERCA DEL ESPAÑOL. II. CAMBIO ADMINISTRATIVO EN PORTUGAL: DE LA PERSISTENCIA INSTITUCIONAL A LA INNOVACIÓN INCREMENTAL. III. EPÍLOGO: ¿Y LA TRANSFORMACIÓN DEL ESTADO EN ESPAÑA? BIEN GRACIAS, PORQUE CINCO DÉCADAS DESPUÉS «SEGUIMOS EN ELLO» ... A PESAR DEL COVID-19. REFERENCIAS BIBLIOGRÁFICAS.
\end{abstract}

\title{
PROEMIO: ESPAÑA 2020, ¿POR QUÉ APENAS HEMOS APRENDIDO TRAS CINCUENTA AÑOS DE REFORMAS DEL ESTADO?
}

Tantos años de cambio administrativo en los países de la OCDE, para continuar en el mismo lugar (Bjur y Caiden, 1978: 359-365; Kickert, 2001: 91-102; OCDE, 1997 y 2006; Pollitt y Bouckaert, 2011). Al menos esa es la sensación que algunos investigadores en ciencia política, ciencia de la administración y gestión pública tenemos en relación con los numerosos y costosos procesos de reforma-modernización administrativa que han tenido lugar en los últimos cincuenta años, entre otros Estados, en nuestra España plural, multinivel e intergubernamental. Y es que, desde hace más de cinco décadas sabemos que hay múltiples factores sociales, políticos y administrativos que justifican estos planteamientos (Baena del Alcázar, 1988; Canales Aliende, 1987; Villoria Mendieta, 1996; Bañón Martínez y Carrillo Barroso, 1997; Ramió Matas, 1999a; Ballart y Ramió Matas, 2000; Olmeda Gómez, Parrado Díez, 1999 y 2000; Crespo González y Pastor Albadalejo, 2002; Varela Álvarez, 2003; Olmeda Gómez, Parrado Díez y Colino Cámara, 2012; Ramió Matas, 2015 y 2017; Arenilla Sáez, 2018: 3.715-3.730). De este marco multidimensional de cambio del Estado que coadyuva a la peculiar "senda de dependencia» institucional española (y que en otros trabajos defino como de «inmovilismo modernizador», Varela Álvarez, 2020: 29-51), destacamos los siguientes factores:

- La incomprensión sobre lo determinante que resulta analizar la historia y el contexto institucional de las transformaciones político-administrativas estatales, con el fin de comprender los cambios, reformas y modernizaciones en sus variados niveles de gobierno-administración;

- la falta de consideración (y gestión) de las diferentes culturas socioeconómicas, así como de sus tradiciones políticas y administrativas, en la construcción de la agenda de cambio del Estado y sus políticas públicas de reforma-modernización administrativa;

- la insistencia en el desarrollo de proyectos de cambio con una orientación juridicista y economicista, como único modelo posible de transformación de las instituciones públicas;

- la persistencia de diferentes tipos de presiones y bloqueos sobre las estructuras administrativas, entre las que destacan los bloqueos políticos, los de los altos cuerpos de la función pública, o los de los sindicatos del sector público;

- la falta de comprensión mutua entre el sector público y el sector empresarial (de las empresas más grandes a las más pequeñas) y social («quangos»), que no acompaña a la complejidad de los escenarios sociales y económicos actuales, así como al necesario impulso de la cooperación institucional, en un proceso del que todos ellos deberían formar parte como colaboradores necesarios para la prestación de servicios públicos.

Para el caso de la España autonómica, multinivel e intergubernamental, estas presiones y bloqueos mencionados provocan, además, una enorme presión neocorporativista público-privada en el conjunto del Estado, así como la ausencia de una cultura de la cooperación y del consenso social, político y administrativo intra, inter y extra institucional (Rojo Salgado y Varela Álvarez, 2013: 337-373; Varela Álvarez y Araújo, 2018). Todo ello, en parte, contribuye al desentendimiento político de una ciudadanía que reclama políticas y servicios públicos, aunque sin implicarse en su control y exigencia de responsabilidades, transparencia y rendición de cuentas, delegando estas acciones en partidos políticos y cargos «politécnicos» (altos cuerpos y directivos públicos) del conjunto de instituciones formales e informales del Estado (Del Campo García, 2012: 191-209).

El breve diagnóstico anterior, repetido hasta la saciedad reforma tras reforma, modernización tras modernización, nos debe llevar a estudiar otros procesos de cambio administrativo de nuestro entorno, con el fin de aprender de otras experiencias de diseño e implementación de políticas públicas de transformación político-administrativas. 
Y esta es la justificación por la cual seleccionamos la experiencia de reforma administrativa de la Administración Central del Gobierno de Portugal, ya que la comprensión de su «senda de dependencia» institucional, sus «luces y sombras», aciertos y errores, buenas y malas prácticas son de mucha utilidad para la formulación, diseño e implementación de procesos de cambio en un Estado como el español. Todas ellas son experiencias, en definitiva, que permiten conocer en profundidad las iniciativas de cambio de un Estado vecino que parte de la misma tradición administrativa que la española.

Porque Portugal, en nuestra opinión, ha conseguido implementar una política pública de modernización administrativa con efectos en algunas de sus dimensiones de organización, como es el caso singular de la gestión de recursos humanos, y en particular de su experiencia institucional en materia de reclutamiento y selección de los directivos de su Administración Central (Varela Álvarez y Mahou Lago, 2005: 1-19; Bouzas Lorenzo y Varela Álvarez, 2005: 61-78; Varela Álvarez y Araújo, 2013: 152-181; Varela Álvarez, Araújo y Briones Gamarra, 2017: 271-292; Varela Álvarez, 2020: 29-51).

De esta manera, en el presente trabajo vamos a analizar el camino marcado por Portugal, teniendo siempre presente la recomendación sabia de quien fue el primer Presidente de la Comissão de Recrutamento para a Administração Pública (CRESAP), y uno de los mayores expertos científicos en el campo de la ciencia de la administración y la gestión pública lusa, João Bilhim (Areses Vidal y Blanco Gaztañaga, 20171); quien recuerda que:

"Las lecciones de la Administración Pública comparada indican que, en caso alguno, se debe exportar/importar modelos acabados de otros países. Ese fue el principal error cometido por los heraldos/misioneros de la Nueva Gestión Pública, oriunda del paradigma administrativo anglosajón. No obstante, podemos y debemos inspirarnos en lo que otros países hacen bien. Así, soy del parecer de que, con las debidas cautelas, la experiencia portuguesa podría ser objeto de inspiración para España.».

En definitiva, sabemos que Portugal y España comparten tradiciones político-administrativas, como también somos conscientes de que la evolución institucional de ambos Estados en materia de reformas y modernizaciones administrativas han sido diferentes, y, en algunos casos, con resultados diametralmente opuestos (Varela Álvarez, 2020). Para poder conocer mejor estas singularidades de cambio administrativo, vamos a desarrollar una presentación sumaria con el objetivo de intentar responder a la pregunta de ¿por qué en España apenas hemos aprendido tras cinco décadas de reformas del Estado? A tal fin, se nos antoja imprescindible presentar el sistema político-administrativo portugués; conocer algunas de las líneas centrales de sus políticas públicas de cambio administrativo; para, finalmente, poder extraer a partir de su experiencia, algunas lecciones que orienten la enésima propuesta de reforma-modernización administrativa del Estado español, ya en la tercera década del siglo XXI, y en plena pandemia sistémica global, regional y local.

\section{EL SISTEMA POLÍTICO-ADMINISTRATIVO PORTUGUÉS, TAN LEJOS TAN CERCA DEL ESPAÑOL}

Portugal es, para algunos españoles, un Estado más en el conjunto de los países que integran la actual Unión Europa. Para otros, es, además, un espacio común de vida cotidiana que se remonta siglos atrás, y que cuenta con una milenaria historia común (Varela Álvarez, 2003: 96-103; Ferreira Rodrígues y García Pérez, 2010; García Pérez y Lobo-Fernandes, 2007; Rojo Salgado, 2007: 235-252; Almuiña Fernández, 2014: 137-146), sobre todo en los espacios fronterizos de ambos estados (López Mira, 2004: 43-50; Cairo Carou, Godinho y Pereiro Pérez, 2009).

Esta es una de las razones, aunque no la única, que nos debe permitir observar con cierto detalle las prácticas institucionales de gobierno de nuestro vecino, más allá de los estereotipos y clichés, como bien nos recuerda Rojo Salgado (2007: 235-252). En mi opinión, merece la pena realizar el esfuerzo de análisis, al menos con la misma intensidad e interés con el que hemos venido estudiando el modelo político-administrativo francés en los últimos dos siglos.

El autor expresa esta opinión en el contexto de una entrevista realizada por Xose ARESES VIDAL y Carmen BLANCO GAZTAÑAGA (2017), "La experiencia portuguesa de la CRESAP: un modelo para España", HayDerecho-Expansión (15 enero 2017 ; accedido el 10 de noviembre del 2020, https://hayderecho.expansion.com/2017/01/15/la-experiencia-portuguesa-de-la-cresad-un-modelo-para-espana/). 
En todo caso, la experiencia portuguesa de reforma del Estado va más allá de las relaciones históricas y de proximidad geográfica («tan lejos, tan cerca», en palabras de Almuiña Fernández, 2014: 137-146). Porque Portugal, como señalaremos más adelante, aporta a la experiencia de cambio institucional una manera diferente de comprensión de las transformaciones de sus administraciones central y municipales (la regionales son una extensión administrativa desconcentrada de Lisboa, a excepción de Açores y Madeira).

Para el análisis de la dimensión político-administrativa que nos ocupa, Portugal se encuentra cerca de nuestra tradición y modelo napoleónico, y muy lejos de las prácticas de reforma administrativa que se han seguido en España a lo largo de los últimos cuarenta años de democracia («caminos paralelos, deficiencias comunes»; Bouzas y Varela, 2005: 61-78). O al menos esto ha sido así hasta la primera década del siglo XXI.

Una lectura pormenorizada de diferentes investigaciones académicas e institucionales de los expertos portugueses, que cubren el período que va desde los inicios del siglo $\mathrm{XX}$, hasta los primeros años de presente siglo (Oliveira, 1991 y 2001; Bilhim, 2000; Araújo, 2000, 2001, 2002a, 2002b, 2013: 25-61; Varela Álvarez, Araújo y Briones Gamarra, 2017: 271-292), nos aporta información relevante para comprender por qué y cómo ha cambiado Portugal su sistema político-administrativo. Y es que, a lo largo de estas décadas, las élites políticas y administrativas portuguesas definen un modelo de organización, funcionamiento y recursos humanos de su Administración Central, caracterizado por (Oliveira, 1991; Araújo, 2002a):

- La construcción de un sistema institucional de corte napoleónico y neocorporativista, vinculado al diseño de su modelo burocrático desde los inicios del siglo XX.

- La consolidación de un sistema burocrático a partir de un marco dictatorial («Estado Novo»).

- La transición a un sistema democrático («Revolução dos Cravos» del 25 de Abril de 1974 y Constitución Portuguesa de 1976), desde las bases jurídicas de la burocracia anterior.

- La adaptación del Estado portugués al sistema de relaciones internacionales, con las consiguientes influencias en su subsistema administrativo (negociación para la entrada en la Comunidad Económica Europea, entre 1974 y 1986).

- La incorporación de los procesos de reforma administrativa globales a la agenda del Estado luso (OCDE y CEE).

- La evolución de una burocracia a una «tecnoburocracia» estatal, con la sustitución de la racionalidad jurídica por la de gestión pública (Oliveira, 1991).

- La «persistencia institucional» de los marcos administrativos estatales a lo largo del siglo XX, a pesar de la implementación de las agendas de reformas y modernizaciones administrativas (Araújo, 2002a).

- La implementación, ya en el presente siglo, de una nueva orientación de cambio administrativo apoyado en una suerte de «innovación incremental» (Varela Álvarez, 2020), impulsada, entre otras causas, por efecto de las presiones político-administrativas de la Unión Europea («Memorandum de Entendimiento» y «Troika»; Varela Álvarez, Araújo y Briones Gamarra, 2017: 271-292).

Como se puede confirmar revisando los factores de cambio del Estado mencionados, Portugal construye su modelo de organización institucional, sus estructuras administrativas, así como sus modelos de gestión a lo largo del pasado siglo. En estas décadas diseña un modelo burocrático de funcionamiento que le ayuda a implementar un sistema de políticas públicas y prestación de servicios públicos que transitan de la modernidad a la contemporaneidad, con agendas de cambio reformistas, muy similares a las españolas.

Sin embargo, es en el último cuarto del siglo XX cuando Portugal emprende las transformaciones institucionales más innovadoras, desarrollando un modelo más próximo a los postulados de la nueva gestión pública y al gerencialismo (Varela Álvarez, Araújo y Briones Gamarra, 2017: 271-292); todo ello, en paralelo a su proceso de transición a la democracia tras la muerte del dictador Salazar. Bien es cierto que existen resistencias y presiones institucionales de las élites políticas y burocráticas a lo largo de estas últimas tres décadas; como es igual de cierto que algunas de esas presiones internas y externas provocan un cambio que alejan a Portugal de España, al menos en la orientación de sus proyectos de cambio administrativo, como vamos a exponer a continuación.

\section{CAMBIO ADMINISTRATIVO EN PORTUGAL: DE LA «PERSISTENCIA INSTITUCIONAL» A LA «INNOVACIÓN INCREMENTAL»}

El cambio administrativo portugués tiene desde hace décadas su base teórica en los enfoques neoinstitucionalistas desarrollados, entre otros, por Powell y Dimaggio, North, Williamson, March y Olsen, Peters, Nohlen, 
O'Donnell o Steinmo. No siendo este el trabajo en cual sea posible desarrollar sus marcos conceptuales ${ }^{2}$, sin embargo, si entendemos necesario acudir a ellos tal y como lo han hecho los investigadores portugueses de la escuela de la Ciencia da Administração y Gestão Pública de la Universidade do Minho desde principios de los años 90 del pasado siglo (Oliveira, 1991 y 2001; Araújo, 2000, 2001, 2002a, 2002b, 2013: 25-61). Sólo así cobra sentido el contexto institucional político-administrativo al que nos hemos referido en el apartado anterior, y que podría sintetizarse en las siguientes cuatro etapas de procesos de reforma-modernización administrativa lusa:

- «Etapa 1974-1985», «La reforma administrativa y la Revolução de Abril de 1974».

- «Etapa 1985-1995», «Revolución managerial de la Administración Pública».

- «Etapa 1996-2002», «La Reinvención de la Administración Pública».

- «Etapa 2002-2005», «La Reingeniería en la Administración Pública».

En estos grandes bloques de representación del cambio institucional portugués aparecen enunciados los programas de transformaciones político-administrativas del último Portugal democrático, al menos, hasta llegar a mediados de la primera década de nuestro siglo, donde el proceso sufre una orientación y aceleración propia que la desmarca, definitivamente, del modelo español de nuestros días.

Porque la aprobación de la Constitución Portuguesa de 1976, representa una nueva manera de «pensamiento institucional» (Heclo, 2010) que, lenta pero inexorablemente, introduce a Portugal en el contexto político y administrativo que viene marcado, entre otros, en las directrices de la OCDE. Es así cuando las influencias, tensiones y presiones de la Comunidad Económica Europea consiguen que nuestro vecino ibérico complemente el modelo de reforma-modernización administrativo de orientación juridicista y economicista clásico, por el propio de la agenda gerencialista de la OCDE.

En este punto la burocracia lusa evoluciona hacia un modelo gerencial, de gestión pública más «neoempresarial» que «neopúblico» (Ramió Matas, 1999b: 65-97), tanto por lo que respecta a los modelos de reinvención del gobierno, como de reingeniería del sector público (Rocha, 1991). Parece claro por los trabajos de Oliveira (1991) y de Soriano García y Bilhim (2013), que pese a todo tiene lugar una «persistencia institucional» (Araújo, 2002) que sólo es vencida por presiones nacionales de todo tipo (sociales, financieras y políticas), así como por las inevitables influencias internacionales fruto de los procesos de modernización administrativa desarrollados en el marco de la OCDE y la Unión Europea (Pollitt y Bouckaert, 2011).

Estas influencias provocan una gran variedad de cambios en diferentes subsectores del Estado que van desde la misma Administración Central, hasta sus periferias administrativas desconcentradas (Comissões de Coordenação e Desenvolvimento Regional, CCDR); así como a los gobiernos locales (autarquías), los cuales a pesar de su reducido número (308 municípios para todo el territorio del Estado), cuentan con un gran peso institucional en el conjunto del sector público, así como una gran relevancia en la prestación de políticas y servicios públicos de bienestar. Todo ello, sin olvidar el hecho de que la inexistencia de un sistema institucional político regional le permite al Estado Central acometer procesos de reformas en todo el territorio sin tener que diseñar un modelo de gobernanza multinivel.

Si entre el año 1974 y el 2005 encontramos en Portugal la construcción de un modelo «buro-gerencial» de reforma administrativa formulado a través de dinámicas isomórficas de diverso signo (miméticas, coercitivas y, en menor medida normativas), el siglo XXI va a marcar un nuevo modelo de cambio impulsado por prácticas de isomorfismo coercitivo. Porque si en los 70 y 80 la influencia de la Comunidad Económica Europea y la OCDE (entre otros) fue clave, en las décadas de 2000 y 2010, y por motivos claramente socioeconómicos, las presiones de la Unión Europea y su «Troika», resultaron definitivos (Varela Álvarez, 2020: 29-51). Los efectos de las políticas financieras de austeridad europea que se ponen en marcha a lo largo de la «década ominosa» (2008 al 2018; Varela Álvarez, Araújo y Briones Gamarra, 2017: 271-292; Varela Álvarez y Araújo, 2018), y que tuvieron su origen en la crisis hipotecaria norteamericana («subprime»), impactan en todo el sistema financiero global, ayudando así a vencer las pocas fuerzas y resistencias que les quedaban a las élites administrativas portuguesas (y que pervivían en su «código genético», Araújo, 2002), así como a su clase sindical (Varela Álvarez, Araújo y Briones Gamarra, 2017: 271-292). Fueron estos, años de intervención exterior en la soberanía económica lusa, intervención que Portugal tuvo que solicitar durante el Gobierno del socialista José Sócrates en el año 2011, y que provocó la pérdida del control de sus cuentas y gastos en todos los niveles de gobierno, a partir de los mecanismos recogidos en los «Memorandos de En-

2 Para ello se puede consultar literatura específica desarrollada por los politólogos españoles desde hace dos décadas, entre ellos: SALVADOR SERNA, 2001; RAMIÓ MATAS y SALVADOR SERNA, 2005; DEL CAMPO GARCÍA, 2012: 191-209; PARRADO DÍEZ, 2015; VARELA ÁLVAREZ y ARAÚJO, 2018. 
tendimiento» de la Unión Europea (CONSEJO UNIÓN EUROPEA, 2010) ${ }^{3}$. Y por aquel entonces, el cambio definitivo acabó por llegar en forma de un modelo peculiar, que he definido en otro trabajo como de «innovación incremental» (Varela Álvarez, 2020: 29-51), y que desborda la «persistencia institucional» característica de estos últimos tiempos de Estado democrático en Portugal.

El modelo de «innovación incremental» de modernización del Estado, supone considerar que es posible desarrollar cambios incrementales, sostenidos, constantes y adaptados a los tiempos de sus propias administraciones públicas, bajo lógicas de «innovación administrativa» (algo que no es necesariamente un oxímoron, como nos recuerdan Ramió Matas y Salvador Serna, 2018: 31-38). La diferencia entre la «innovación incremental» y la «innovación radical», siguiendo a García y Calantone (2002: 110-132), reside en el hecho de que la primera estaría relacionada con la mejora de una organización (posición, producto, organización); mientras que la segunda, debería vincularse a una estrategia de cambios transversales y holísticos en el conjunto del entorno en el que opera esta organización. Para nosotros, la «innovación incremental» desarrollada por el Estado portugués en sus instituciones públicas, implica la introducción de prácticas isomorfistas de todo tipo en el conjunto de su gobierno-organización, siempre con el objetivo puesto en la producción de impactos estratégicos en el contexto de su mapa de ecología institucional, empresarial y social.

En el fondo, este proceso no es más que la adaptación virtuosa del Estado portugués a una intervención internacional absoluta de su sistema social, económico, político, y, por ende, administrativo. Es en este estado de evolución institucional, cuando ni siquiera la ciudadanía, el sector empresarial y el tercer sector pueden mantenerse ajenos a un proceso holístico y transversal de cambio. La política de modernización administrativa deja de ser una «ocurrencia» de la clase política, o un diseño «lampedusiano» de la élite burocrática pública, para convertirse en un proyecto de Estado, supervisado, eso sí, por la Comisión Europea, el Banco Central Europeo y el Fondo Monetario Internacional.

Porque del 2005 al 2019 (siempre antes del impacto que el COVID-19 está dejando en los modelos de organización institucionales globales y locales), Portugal adapta sus pre-democráticas estructuras de cambio burocráticas (Secretariado de Reforma Administrativa, SRA, 1968), a otras más contemporáneas insertadas en las diferentes políticas y programas públicas de modernización administrativa, entre las que se pueden destacar el Plano Nacional de Desburocratização (1986), el Programa Integrado de Formação para a Modernização da Administração Pública (1993-1999) o el transversal Sistema Integrado de Gestão e Avaliação do Desempenho na Administração Pública (SIADAP, 2007).

En definitiva, la orientación del cambio del Estado portugués que hemos denominado como «innovación incremental», potenciada por los mecanismos de isomorfismo coercitivo de la «Troika», desbordan las clásicas orientaciones incrementalistas basadas en prácticas isomorfistas miméticas y normativas del siglo XX. A pesar de todo, los modelos clásicos «buro-gerenciales» siguen presentes en el «ADN» luso porque la agenda global de reformas del Estado se sostiene, aunque con los ajustes propios provocados por los errores «austericidas» cometidos por esa misma «Troika» a lo largo de la década pasada.

La actualidad de las transformaciones del Estado portugués, ya en plena época COVID-19, pasa por adaptar sus instituciones públicas a los marcos financieros que la Unión Europea facilita a través de la estrategia «Next Generation EU» de la Comisión Europea (2020)4, y sus diversos mecanismos de apoyo a la recuperación económica de los Estados miembros. En esta línea, la orientación de la modernización del Estado sigue la senda de la digitalización (en el marco de la Agenda Digital Europea desde el año 2010), que parte del programa SIMPLEX impulsado por su Agência para a Modernização Administrativa (AMA), y que pretende reforzar los ámbitos del «e-Government» y el «teletrabajo».

Tras este breve recorrido por la experiencia de modernización administrativa del Estado en Portugal, resta buscar algunas lecciones para el conjunto de instituciones centrales y autonómicas del Estado español.

\section{EPÍLOGO: ¿Y LA TRANSFORMACIÓN DEL ESTADO EN ESPAÑA? BIEN GRACIAS, PORQUE CINCO DÉCADAS DESPUÉS «SEGUIMOS EN ELLO» ... A PESAR DEL COVID-19}

A finales del año 2020 comenzamos a percibir que las bases institucionales sobre las que se fundan nuestros Estados de bienestar no eran tan sólidas como creíamos, porque el coronavirus SARS-CoV-2 (o

3 Reglamento del Consejo Unión Europea n. ${ }^{\circ}$ 407/2010 de 11 de mayo, que establece el Mecanismo Europeo de Estabilización Financiera, EFSM y del European Financial Stability Facility.

4 Ver https://ec.europa.eu/info/strategy/recovery-plan-europe_es\#nextgenerationeu (accedido, 30-XI-2020). 
COVID-19) nos ha mostrado la debilidad de nuestras administraciones públicas multinivel. En este sentido, y recordando el cuento de Hans Christian Andersen, soy de la opinión de que la sociedad, la política y la economía española, al igual que el conjunto de sus niveles de gobierno-administración, no deberían hacer otra cosa que reconocer su desnudez.

Todo ello a pesar de que disponemos de las ciencias (incluidas las sociales y las jurídicas), a partir de las cuales deberíamos ser capaces de basar nuestros modelos de toma de decisiones y comportamiento político-administrativo en evidencias, en estudios teóricos, en informes de instituciones internacionales de alto nivel. Porque es indudable que las ciencias sociales (y entre ellas la Ciencia de la Administración y la Teoría de la Organización) nos han venido mostrando desde hace décadas (al menos desde los años 70 del siglo $\mathrm{XX}$ ) las posibilidades y limitaciones de los diferentes modelos de Estados, de sus marcos teórico-conceptuales, contextos globales y locales, procesos de cambio, políticas de reforma-modernización y enfoques de gestión pública burocráticos, gerenciales y de gobernanza (y aquí recordamos con especial cariño a Joan Prats i Catalá y sus trabajos sobre este tema; Prats i Catalá, 2005).

Pese al bagaje científico acumulado, somos de la opinión de que apenas hemos aprendido sobre lo que debemos y tenemos que emprender como «Estado administrativo». Al menos esa es la evidencia científica que se puede constatar tras revisar el panorama de los procesos de reforma-modernización españolas desde entonces, hasta la fecha, ya en la tercera década del siglo XXI en plena pandemia sistémica global, regional y local.

Porque si de algo se puede tildar a la experiencia española de modernización administrativa en nuestra historia democrática más reciente, es de un cierto «inmovilismo modernizador». Esta expresión no es más que un oxímoron que muestra lo profundo que ha calado el modelo de experiencias basadas en un peculiar estilo de transformación del Estado, que no es otro que el modelo «lampedusiano» que proclama cambiar toda la administración, para que toda ella (desde la central, hasta las autonómicas y locales) siga igual.

La cuestión es que, lejos de asumir el reto de una transformación racional (legal, económica y de gestión pública) y basada en evidencias científicas, así como en experiencias comparadas adaptadas a nuestra realidad político-administrativa (recordamos las palabras de Bilhim y el informe de la OCDE del año 2006), los responsables «politécnicos» de nuestras instituciones públicas se empeñan en repetir lógicas «burogerenciales» apoyadas estrategias jurídico-formales y de corte economicista. Lo que se puede comprobar en el reciente "Real Decreto-ley 36/2020, de 30 de diciembre, por el que se aprueban medidas urgentes para la modernización de la Administración Pública y para la ejecución del Plan de Recuperación, Transformación y Resiliencia (BOE-A-2020-17340)", que el Gobierno de España ha aprobado a finales del 2020, en el marco del plan de "Plan de Recuperación, Transformación y Resiliencia de la Economía Española" y del plan "Next Generation EU" de la Comisión Europea.

Y es que cinco décadas después (o más, si contamos las experiencias de reforma administrativa franquista de finales de los años 50 del pasado siglo), y en relación a la transformación del Estado en España, podemos confirmar que «estamos bien» porque «seguimos en ello». Eso sí, bajo idénticas lógicas y estrategias y sin alterar nuestro «código genético» administrativo.

¿Podríamos plantear otras alternativas a esta clásica orientación del cambio administrativo español? ¿Sería posible aprender de otras experiencias, teniendo sumo cuidado en no realizar un «copy paste» y adaptándolas a nuestra cultura social, económica, política y, por ende, administrativa?

La Ciencia de la Administración (y las ciencias de management público) así nos lo demuestra. Y no hay que viajar muy lejos para descubrir que, en nuestro espacio peninsular compartido con Portugal encontramos «buenas prácticas» (algunas no tanto) de modernización administrativa de las que es posible extraer algunas lecciones para el conjunto de los gobiernos-administraciones multinivel españoles. A continuación, enunciamos algunas extraídas del análisis desarrollado en las páginas anteriores:

- La primera y más relevante es que o los «policy makers» españoles de todos sus niveles de gobierno comprenden que hay seguir los trabajos de las ciencias sociales (entre otras), así como a sus métodos de análisis comparado y de caso, o no hay política pública de modernización que pueda pasar de la fase de formulación y diseño, a la de implementación ... y no digamos ya a la de evaluación y auditoría pública de sus medidas. Portugal lo ha hecho así, de manera lenta, adaptativa y sostenible, para lo que ha creado (e invertido mucho presupuesto) una base científica solvente e internacional, en torno a sus Universidades públicas desde los años 90 del pasado siglo.

- La segunda pasa por estudiar el contexto global, las experiencias internacionales, pero con una mirada crítica local. Hay que pensar en local, observando lo global (justo al revés del aserto ecologista 
de los años 90), para que los proyectos de cambio administrativo estén alineados con las necesidades de los ciudadanos, del tejido productivo, de su estructura social y societaria, en definitiva. En esto los proyectos modernizadores españoles han mirado siempre hacia el exterior, hacia los modelos globales de cambio ... aunque sólo desde una perspectiva jurídico-formal. Así, en España se ha construido una «realidad administrativa» (Beltrán Villalva, 1991), que no ha conseguido superar los bloqueos políticos y administrativos de sus élites «partitocráticas», burocráticas y sindicales. Portugal, de nuevo, ha conseguido superar parcialmente estos bloqueos («persistencias institucionales»), generando una cultura administrativa que ha ido evolucionando de modelos burocráticos a «tecnoburocráticos» a lo largo de los últimos cuarenta años. Y ya no cabe duda de que la adaptación a los cambios provocados por las presiones internacionales ha resultado clave para la reforma del conjunto de su Estado.

- En línea con el proceso anterior, la tercera lección tiene que ver con los sujetos del cambio administrativo: las personas, los empleados públicos que (sobre)viven (y muchas veces sufren) estos proyectos de reforma-administración; más que participar «en» y «de» ellos. Y es que sin alterar el "código genético» de sus empleados públicos y sin introducir la racionalidad de la gestión pública en los procesos de cambio administrativo no hay posibilidad «real» de pasar de una propuesta en un Real Decreto-Ley («real-administrativa») a un proyecto de gobernanza pública («real-real»; Varela Álvarez y Araújo, 2018), de innovación política, administrativa y social (Lapuente Giné y Suzuki, 2020), así como de desarrollo efectivo de sus líneas estratégicas (no sólo de economía, eficacia y eficiencia viven las instituciones públicas). Portugal ha tomado ese rumbo de cambio de cultura organizativa, bien es cierto que a partir de sucesivos procesos de «isomorfismo coercitivo», y en un largo período de tiempo que va desde los años 80 hasta su intervención por parte de la «Troika» en los inicios de la década pasada. El ejemplo de la CRESAP y su modelo de reclutamiento y selección profesional y meritocrática de directivos y gestores públicos es un buen ejemplo de ello (Varela Álvarez, 2020: 29-51). En todo caso, debe quedar claro que Portugal no cambia su administración «ni por decreto», ni «en un día», ni en todos los niveles de gobierno-administración por igual.

- La última lección que se podría extraer de la experiencia portuguesa, y no para copiar si no para inspirar los procesos propios de cambio administrativo post-COVID-19, debería pasar por entender de manera transversal el concepto de cooperación institucional, y sus complejos procesos de colaboración y coordinación, tanto con el conjunto de la red interinstitucional de un Estado (multinivel o no), como con el conjunto de actores sociales y empresariales de nuestro país (Varela Álvarez y Araújo, 2018). En Portugal este aprendizaje institucional se realiza en diferentes fases, bajo presiones enormes de actores internacionales y en un proceso de europeización similar al español. Bien es cierto que el Estado portugués comprende, asume e interioriza esta necesidad de considerar que el Estado es una parte del sistema, y que su papel como estratega de una red intergubernamental sin actores regionales político-administrativos va a resultar clave para mejorar el progreso colectivo. En España, a sus sectores económicos, sociales y élites administrativas y sindicales, aún les queda por entender esta necesidad de transitar de un modelo burocrático a uno de gobernanza en red efectivo, haciendo funcionar las alianzas más allá de los «Reales Decretos» y de las «subvenciones públicas dirigidas a sectores empresariales», y consiguiendo superar los problemas que las experiencias que los partenariados público-privados han dejado en el panorama institucional español de los últimos años. Sin duda, el reto de las alianzas y los partenariados impulsados por la Agenda 2030 y los Objetivos de Desarrollo Sostenible, así como por el proyecto de la Comisión Europea «Next Generation EU», tendrá que pasar por convertir las miradas y vetos cruzados socioeconómicos y políticoadministrativos, en otras prácticas basadas en modelos de cooperación y desarrollo de consensos ampliados a una base social y ciudadana, que debe participar de este proceso de modernización post-COVID-19 de una manera comprometida y responsable, superando las clásicos estilos de acción colectiva institucional basados en la participación formal en las páginas web institucionales, los foros de consultas técnicas, o los espacios de concertación formales y de redes sociales.

Las lecciones extraídas de la extensa experiencia portuguesa no implican la realización de grandes proyectos de inversión económica; tampoco de enormes e inacabables desarrollos normativos y legislativos sobre «medidas urgentes» de modernización del Estado. De esto está sobrada la experiencia española de modernización administrativa con procesos del tipo «stop \& go», y sin dirección clara (o en el peor de los casos, en círculo). Porque más que crear Agencias, para suprimirlas después, o sustituirlas por Autoridades 
DA. Nueva Época - N. ${ }^{\circ}$ 7, enero-diciembre 2020 - ISSN: 1989-8983 - DOI: https://doi.org/10.24965/da.i7.10897 - [Págs. 145-155]

La experiencia de la modernización administrativa en Portugal. Algunas lecciones para las instituciones públicas..

Enrique José Varela Álvarez

Independientes, el cambio que está por llegar a las instituciones pública españolas pasa por comprender que o se produce una auténtica transformación de la cultura social, económica, política y administrativa en relación con el sentido de las instituciones públicas, o la transformación del Estado tendrá que seguir esperando, una vez más, a pesar de la emergencia colectiva provocada por la pandemia del COVID-19.

Nadie mejor que el premio Nobel José Saramago, para entender que lo que podemos aprender los españoles de los portugueses en un contexto de crisis (como el que recoge en su magistral libro "A Jangada de Pedra"), tiene que ver con la capacidad de adaptación conjunta como colectividad ante las adversidades. Eso es lo que nos enseña Portugal sobre su «senda institucional» de modernización administrativa.

Eso, ni más ni menos, es lo que le queda a España para conseguir, de una vez por todas. Y estoy convencido de que sólo se logrará provocando un cambio cultural político-administrativo que nos sitúe en un marco profesional y meritocrático superador de las malas prácticas burocráticas y neoburocráticas de los siglos XIX y XX; marco en el que la ciudadanía y los sectores sociales y empresariales se vean reflejados para superar de manera conjunta esta crisis, y las que estén por llegar.

\section{BIBLIOGRAFÍA}

ALMUIÑA FERNÁNDEZ, C. J. (2014): “España y Portugal: tan cerca geográficamente y tan lejos históricamente”, en La Albolafia: Revista de Humanidades y Cultura, núm. 1, págs. 137-146. http://albolafia.com/trab/Alb-Doss-001. ALMUINA.pdf.

ARAÚJO, J. F. (2013): "Da Nova Gestão Pública à Nova Governação Pública: pressões emergentes da fragmentação das estruturas da Administração Pública”, en SILVESTRE, H.; ARAÚJO, J. F. (eds.): Coletânea em Administração Pública. Lisboa: Escolar Editora.

ARAÚJO, J. F. (2002a): Gestão Pública em Portugal: mudança e persistencia institucional. Coimbra: Quarteto Editora.

ARAÚJO, J. F. (2002b): "NPM and the Change in Portuguese Central Government", en International Public Management Journal, vol. 5, núm. 3, págs. 223-236. http://repositorium.sdum.uminho.pt/bitstream/1822/5844/1/ NPM\%20and\%20the\%20change\%20in\%20portuguese\%20central\%20government.pdf.

ARAÚJO, J. F. (2001): "Considerações sobre o conceito de reformas administrativa", en Revista de Administração e Políticas Públicas, vol. II, núm. 2, págs. 60-63.

ARAÚJO, J. F. (2000): "Tendências Recentes de Abordagem à Reforma Administrativa”, en Revista da Associação Portuguesa de Administração e Políticas Públicas, vol. I, núm. 1, págs. 38-47.

ARENILLA SAÉZ, M. (2018): "Avances y resistencias en la modernización administrativa española desde 1978”, en PENDÁS GARCÍA, B. (dir.), GONZÁLEZ HERNÁNDEZ, E.; RUBIO NÚÑEZ, R. (coords.): España constitucional (1978-2018): trayectorias y perspectivas, vol. 5, tomo 5. págs. 3.715-3.730. Madrid: Centro de Estudios Políticos y Constitucionales

ARESES VIDAL, X.; BLANCO GAZTAÑAGA, C. (2017): "La experiencia portuguesa de la CRESAP: un modelo para España", HayDerecho-Expansión (15 enero 2017; accedido el 10 de noviembre del 2020). https://hayderecho. expansion.com/2017/01/15/la-experiencia-portuguesa-de-la-cresad-un-modelo-para-espana/.

BAENA DEL ALCÁZAR, M. (1988): Curso de Ciencia de la Administración, vol. I. Madrid: Tecnos, 2 . $^{\text {e ed. }}$

BALLART, X.; RAMIÓ MATAS, C. (2000): Ciencia de la Administración. Valencia: Tirant lo Blanch.

BAÑÓN MARTÍNEZ, R.; CARRILLO BARROSO, E. (1997): La nueva Administración Pública. Madrid: Alianza Editorial.

BELTRÁN VILLALVA, M. (1991): La realidad social. Madrid: Editorial Tecnos.

BILHIM, J. (2000): Ciência da Administração. Lisboa: Universidade Aberta.

BJUR, W.; CAIDEN, G. (1978): "On Reforming Institutional Bureaucracies", en International Review of Administrative Sciences, vol. 44, págs. 359-365. https://doi.org/10.1177/002085237804400405.

BOUZAS LORENZO, R.; VARELA ÁLVAREZ, E. (2005): "Caminos paralelos, deficiencias comunes: aproximación a los modelos públicos de recursos humanos en España y Portugal”, en Gestión y Análisis de Políticas Públicas (GAPP), núm. 32, págs. 61-78. https://doi.org/10.24965/gapp.vi32.384.

CAIRO CAROU, H.; GODINHO, P.; PEREIRO PÉREZ, X. (2009): Portugal e Espanha. Entre discursos de centro e práticas de frontera. Lisboa: Edições Colibri.

CANALES ALIENDE, J. M. (1987): Panorama actual de la Ciencia de la Administración. Madrid: Instituto Nacional de Administración Pública.

CRESPO GONZÁleZ, J.; PASTOR ALBADALEJO, G. (2002): Administraciones Públicas Españolas. Madrid: McGraw-Hill.

DEL CAMPO GARCÍA, E. (2012): "Instituciones y gobernanza en América Latina: la continuidad de las reglas informales”, en BARREDA DÍEZ, M.; CERRILLO I MARTÍNEZ, A. (coords.): Gobernanza, Instituciones y Desarrollo. Homenaje a Joan Prats. Valencia: Tirant lo Blanch.

FERREIRA RODRÍGUES, T.; GARCÍA PÉREZ, R. (2010): Portugal e Espanha. Crise e convergência na União Europeia. Lisboa: Editorial Tribuna de Historia. 
DA. Nueva Época - N. ${ }^{\circ}$ 7, enero-diciembre 2020 - ISSN: 1989-8983 - DOI: https://doi.org/10.24965/da.i7.10897 - [Págs. 145-155]

La experiencia de la modernización administrativa en Portugal. Algunas lecciones para las instituciones públicas..

Enrique José Varela Álvarez

GARCÍA, R.; CALANTONE, R. (2002): "A critical look at technological innovation typology and innovativeness terminology: A literatura review", en Journal of Product Innovation Management, vol. 19, págs. 110-132. https://doi. org/10.1016/S0737-6782(01)00132-1.

GARCÍA PÉREZ, R.; LOBO-FERNÁNDES, L. (2007): España y Portugal. Veinte años de integración europea. Santiago de Compostela: Tórculo Ediciones.

HECLO, H. (2010): Pensar institucionalmente. Barcelona: Editorial Paidós.

KICKERT, W. J. M. (2001): "Public management reforms in western governments", en VV.AA: A Administração Pública no limiar do Século XXI: os grandes desafíos, págs. 91-102. Oeiras: INA.

LAPUENTE GINÉ, V.; SUZUKI, K. (2020): "Politicization, Bureaucratic Legalism, and Innovative Attitudes in the Public Sector", en Public Administration Review, vol. 8, núm. 3, págs. 454-467. https://doi.org/10.1111/puar.13175.

LÓPEZ MIRA, Á. X. (2004): "Galaecos españois e porto galaecos: Identidades e fronteiras", en Revista Pensamento do Eixo Atlántico, núm. 6, págs. 43-50.

OCDE (2006): La modernización del Estado: El camino a seguir. Madrid: Instituto Nacional de Administración Pública. https://doi.org/10.1787/9789264030305-es.

OCDE (1997): La transformación de la gestión pública. Las reformas en los países de la OCDE. Madrid: BOE-MAPMinisterio de Presidencia.

OLIVEIRA, J. A. (1991): Princípios de Gestão Pública. Lisboa: Editorial Presença. 1. a ed.

OLIVEIRA, J. A. (2001): Gestão Pública e Modernização Administrativa. Oeiras: INA.

OLMEDA GÓMEZ, J. A. (1999): Ciencia de la Administración. Volumen I. Teoría de la Organización y Gestión Pública. Madrid: UNED.

OlMEdA GÓMEZ, J. A.; PARRADO DÍEZ, S. (2000): Ciencia de la Administración. Volumen II. Los Sistemas Administrativos. Madrid: UNED.

OLMEDA GÓMEZ, J. A., PARRADO DÍEZ, S.; COLINO CÁMARA, C. (2012): Las Administraciones Públicas en España. Valencia: Tirant lo Blanch.

PARRADO DÍEZ, S. (2015): El análisis de la gestión pública. Valencia: Tirant lo Blanch. 1. a ed.

PRATS I CATALÁ, J. B. (2005): De la burocracia al «management», del «management» a la burocracia. Las transformaciones de las Administraciones Públicas de nuestro tiempo. Madrid: Instituto Nacional de Administración Pública.

POLLITT, CH.; BOUCKAERT, G. (2011): Public Management Reform. A comparative analysis - new public management, governance and the neo-weberian State. Oxford: Oxford University Press. $3 .^{a}$ ed.

RAMIÓ MATAS, C. (2015): Administración Pública y Crisis Institucional. Estrategias de reforma e innovación para España y América Latina. Madrid: Tecnos. 1. a ed.

RAMIÓ MATAS, C. (2017): La administración pública del futuro (Horizonte 2050). Instituciones, política, mercado y sociedad de la innovación. Madrid: Tecnos. 1. ${ }^{\text {a }}$ ed.

RAMIÓ MATAS, C. (1999a): Teoría de la Organización y Administración Pública. Madrid: Tecnos; Barcelona: Universitat Pompeu Fabra.

RAMIÓ MATAS, C. (1999b): "Corrientes Neoempresariales versus Corrientes Neopúblicas: Cultura Administrativa, Valores Públicos y Credibilidad Social. Un Planteamiento Radical”, en Instituciones y Desarrollo, núm. 5, págs. 65-97.

RAMIÓ MATAS, C.; SALVADOR SERNA, M. (2018): "Innovación en las Administraciones Públicas: ¿Un oxímoron o una realidad?", en Consultor de los Ayuntamientos. núm. extra 1 (mayo), págs. 31-38.

RAMIÓ MATAS, C.; SALVADOR SERNA, M. (2005): Instituciones y nueva gestión pública en América Latina. Barcelona: Fundaciò CIDOB.

CONSEJO DE LA UNIÓN EUROPEA (2010): Reglamento 407/2010, de 11 de mayo, que establece el Mecanismo Europeo de Estabilización Financiera. Diario oficial de la Unión Europea, L118, de 12 de mayo de 2010. https:// eur-lex.europa.eu/legal-content/ES/TXT/HTML/?uri=CELEX:32010R0407\&from=ES.

ROJO SALGADO, A. (2007): "Percepciones mutuas e imágenes recíprocas: España y Portugal ante el espejo", en GARCÍA PÉREZ, R.; LOBO-FERNÁNDES, L. (coords.): España y Portugal. Veinte años de integración europea, págs. 235-252. Santiago de Compostela: Tórculo Ediciones.

ROJO SALGADO, A.; VARELA ÁLVAREZ, E. J. (2013): "La buena gobernanza del Estado Autonómico: un largo camino por recorrer", en FERRI DURÁ, J. (comp.): Política y Gobierno en el Estado Autonómico. Valencia: Tirant lo Blanch.

SALVADOR SERNA, M. (2001): "El Papel de las Instituciones en la Gestión de las Administraciones Públicas", en Revista del CLAD Reforma y Democracia, núm. 20, págs. 73-108.

SORIANO GARCÍA, J. E.; BILHIM, J. (2013): "La racionalidad política y técnica en la selección de la alta administración pública y gestión pública portuguesa: una experiencia útil para España”, en El Cronista del Estado Social y Democrático de Derecho, núm. 38, págs. 36-52.

STEINMO, S. (2013): "Institucionalismo histórico", en DELLA PORTA, D.; KEATING, M. (eds.): Enfoques y metodologías de las ciencias sociales: una perspectiva pluralista, págs. 131-152. Madrid: Editorial Akal.

VARELA ÁLVAREZ, E. J. (2020): “¿Es la CRESAP el modelo institucional de selección adecuado para profesionalizar la función directiva en España? Puede que sí, puede que no", en Revista de Administração e Emprego Público, núm. 6 , págs. 29-51. 
VARELA ÁlVAREZ, E. J. (2003): Administraciones Públicas Contemporáneas en España. Una Visión Multidisciplinar desde el Estado de las Autonomías. Santiago de Compostela: Tórculo Edicións.

VARELA ÁlVAREZ, E. J.; ARAÚJO, J. F. (2018): Cooperación Institucional o ¿Cómo gestionar redes y partenariados en el ámbito público? Valencia: Tirant lo Blanch; Madrid: INAP.

VARELA ÁlVAREZ, E. J.; ARAÚJO, J. F. (2013): "La Dirección Pública Profesional en España y Portugal: Un Análisis Neoinstitucional”, en Revista Gestão \& Políticas Públicas, núm. 1, vol. 3, págs. 152-181.

VARELA ÁlVAREZ, E. J.; FERRAZ ESTEVES DE ARAÚJO, J. F.; BRIONES GAMARRA, Ó. (2017): "Civil Service and the Crisis: A Comparative Analysis of Iberian Countries (2008-2013)", en SCHOFIELD, N.; CABALLERO, G. (eds.): State, Institutions and Democracy. Studies in Political Economy. Cham: Springer International Publishing. https://doi.org/10.1007/978-3-319-44582-3_12.

VARELA ÁLVAREZ, E. J.; MAHOU LAGO, X. M. ${ }^{a}$ (2005): "Reforma de la administración pública central en Portugal: governance, recursos humanos y nuevas tecnologías", en X Congreso Internacional del CLAD sobre la Reforma del Estado y de la Administración Pública, págs. 1-19. Santiago, Chile, 18-21 Oct. 2005.

VILLORIA MENDIETA, M. (1996): La modernización de la administración como instrumento al servicio de la democracia. Madrid: BOE; INAP. 